\title{
A Layered Fuzzy Controller For Nonholonomic Car-Like Robot Motion Planning
}

\author{
Mohamed M. El-Khatib \\ Electronic and Electrical Engineering Dpt. \\ University of Strathclyde \\ 204 George St., G1 1XW, Glasgow \\ United Kingdom \\ melkhatib@eee.strath.ac.uk
}

\author{
David J. Hamilton \\ Electronic and Electrical Engineering Dpt. \\ University of Strathclyde \\ 204 George St., G1 1XW, Glasgow \\ United Kingdom \\ d.hamilton@eee.strath.ac.uk
}

\begin{abstract}
A system for real time navigation of a nonholonomic car-like robot in a dynamic environment consists of two layers is described: a Sugeno-type fuzzy motion planner; and a modified proportional navigation based fuzzy controller. The system philosophy is inspired by human routing when moving between obstacles based on visual information including right and left views to identify the next step to the goal. A Sugeno-type fuzzy motion planner of four inputs one output is introduced to give a clear direction to the robot controller. The second stage is a modified proportional navigation based fuzzy controller based on the proportional navigation guidance law and able to optimize the robot's behavior in real time, i.e. to avoid stationary and moving obstacles in its local environment obeying kinem atics constraints. The system has an intelligent combination of two behaviors to cope with obstacle avoidance as well as approaching a target using a proportional navigation path.
\end{abstract}

The system was simulated and tested on different environments with various obstacle distributions. The simulation reveals that the system gives good results for various simple environments.

\section{INTRODUCTION}

The motion planning problem involves formulating a trajectory for a given object or system of objects, such that predefined geometric and/or non-geometric constraints are satisfied. These problems have attracted considerable attention in recent years because they are faced in a wide variety of applications, particularly in robotics navigation, autonomous systems, electric packaging, NC machining, guidance and traffic control [1-4].

In its most general form, the motion planning problem is stated as follows:

"Given a system of objects, a start and a goal configurations for these objects, and set of obstacles which can be stationary or moving, the objective is to find the best feasible trajectory for the system of objects to move from the start configuration to the goal configuration such that collision with the obstacles is avoided" [5]

The motion planning problem can be classified as static or dynamic, depending on the mode in which the obstacle information is available. In a static problem, the traditional mode of motion planning, all the information about obstacles and the geometry of the robot in the environment are known a priori, and the motion of the robot is designed from such information. On the other hand, with dynamic planning, only partial information is available about the obstacles and to achieve a given goal; the robot plans a path based on the available information [6], [7]

The main problem when navigating realistic environments is the pervasive uncertainties in the overall system (which includes the robot and surrounding domain). Thus it becomes impossible to generate complete or exact models of the system and/or its behavior. Uncertainties also propagate through the control systems leading to further inaccuracies or errors. By contrast, humans cope very well with uncertain environments, often relying on approximate or qualitative data and reasoning to make decisions and to accomplish their objectives. Therefore artificial intelligent systems which use an approximate reasoning are highly desirable [8-11]. Fuzzy logic systems are inspired by the human capability to operate on, and reason with, perception based information [12]. Fuzzy logic provides a formal methodology for representing and implementing the human expert's heuristic knowledge and perception based actions [13].

The focus of this paper is the real time navigation of a nonholonomic car-like robot in a dynamic environment. The nonholonomic constraints for this system arise from constraining each pair of wheels to roll without slipping. They can only move forward or backward while making a curve with a bounded radius, in the same way as real cars [14], [15]. These constraints imposed on the motion are not integrable and, as a result can not be stabilized by smooth, static feedback controls [16]. Therefore the techniques of discontinuous feedback control, dynamic feedback linearization [17], sliding mode control [18], and fuzzy/neural control [19], [20], have been used to solve stabilization, trajectory tracking and robust control problems. Car-like robots are particularly interesting for the present approach because of the restricted set of motions. This method plans forward motions for the robot (backward motions are only used for backtracking).

\section{II $\quad$ SYSTEM DESCRIPTION AND PROBLEM FORMULATION}

Intelligent Robotic Systems should take task-level commands directly without any planning-type decomposition [18]. Additionally it is desirable to design them for a large class of tasks rather than a specific task [21]. As a result, the spilt between robot controller design and robot action planning is critical since they usually have two different reference bases. The action planning is 
carried out in terms of events, whereas the execution of planned actions uses a reference frame on existing robot control system using a time based or clocked trajectory [8]. Thus, the proposed system consists of two subsystems, a fuzzy motion planner (FMP) and a modified proportional navigation (PN) based fuzzy controller Fig.(2). First a definition of car-like robot kinematics is introduced.

Let $(x, y, \theta)$ denote the configuration of the car-like robot Fig.(1) parameterized by the location of the front wheels. The kinematic model [14], [15] can be represented as:

$$
\begin{aligned}
& \dot{x}=v \cos \theta \\
& \dot{y}=v \sin \theta \\
& \dot{\theta}=\frac{v}{l} \tan \varphi
\end{aligned}
$$

where:

V the forward velocity of the car-like robot

$\theta \quad$ the angle of the vehicle body with respect to the horizontal line

$\varphi \quad$ the steering angle with respect to the vehicle body

$(\mathrm{x}, \mathrm{y})$ the location of the centre point of the front wheels

$\mathrm{L}$ the length between the front and rear wheels

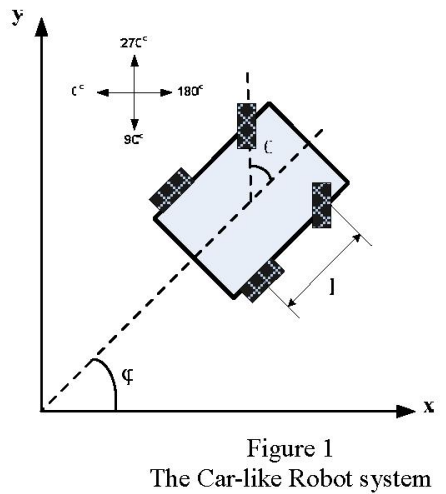

The nonholonomic constraints for this system arise from constraining each pair of wheels to roll without slipping.

In the proposed system Fig.(2), the data from the three sensors plus the direction to the goal are given to the motion planner; the result is a clear direction to move in. Next, the fuzzy controller takes the hand to steer the robot to the goal using the proportional navigation trajectory and avoiding the obstacles.

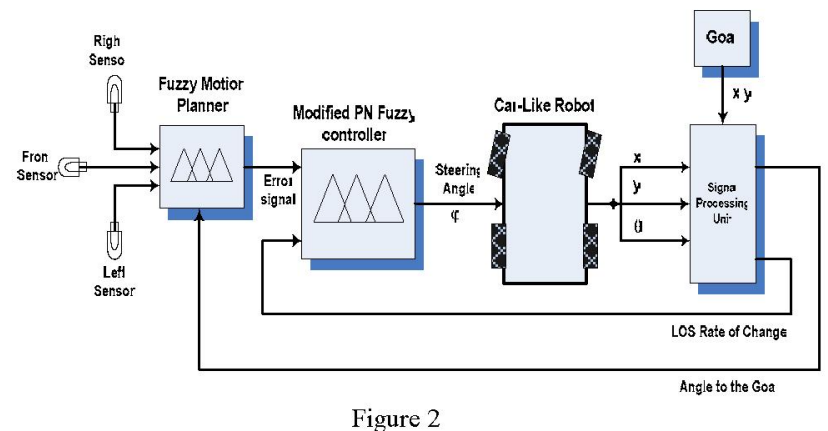

The complete system of fuzzy motion planner and behavioral fuzzy controller.

\section{SUGENO-TYPE FUZZY MOTION PLANNER}

The system philosophy is inspired by human routing when moving between obstacles based on visual information including right and left views to identify the next step to the goal in free space. This is analogous to the proposed robot moving safely in an environment based on data "visible" with three ultrasound range finders. These sensors are mounted to the front, right and left of the robot as shown in Fig.(3).

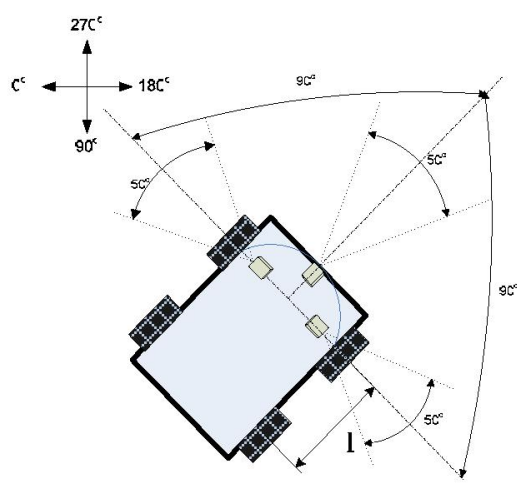

Figure 3

The Robot Sensors with their beam pattern

The Proposed sensors are modeled using Matlab software such that they scan an array of zeros representing the clear environment with obstacles set to ones if present. They scan the array in a $50^{\circ}$ sector along each sensor axis direction such that it returns the range of the nearest obstacle as shown in fig (3). The maximum range is set to be 5 meter. These specifications model commercial sensors such as Ultrasonic range finder SRF04, SRF05 and SRF10 [22].

A Sugeno-type [23], [24], fuzzy motion planner of four inputs one output is introduced to give a free direction to the robot controller. The inputs are the frontal, right and left obstacle ranges found by the front, right and left sensors. The last input is the angle to the goal, which indicates the difference in direction between the goal and the robot's current direction. The output is an error angle representing the angle needed to go from the robot's direction to a free (suggested) direction. Fuzzy membership functions are assigned to each input as shown in Fig.(4).
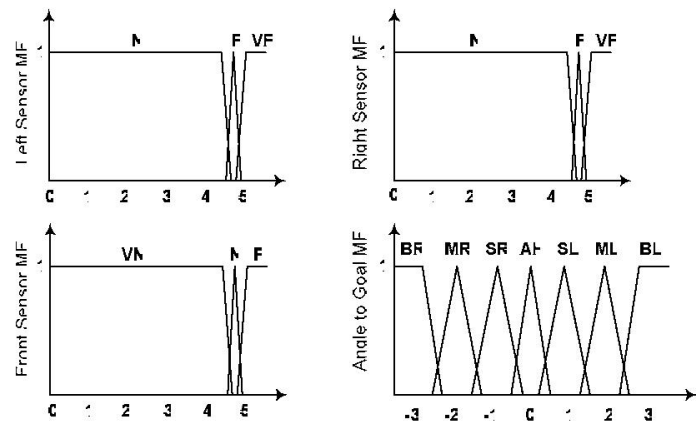

Figure 4

The Membership functions of the fuzzy motion planner inputs

The membership functions describe the ranges from the sensors designed to give the planner early warning. The 
left and right sensors assign the obstacle as $\mathrm{N}$ (near) or VN (very near) for the front sensor if its range approaches $90 \%$ of the sensor maximum range. For example: the obstacle is near if its range is about 4.5 meter as the sensor maximum range is 5 meter. This enables the planner to determine the feasible trajectory at an early stage to avoid cul-de-sacs.

The fuzzy motion planner uses Takagi-Sugeno fuzzy inference for the rule evaluation [23], [24]. Unlike other fuzzy control methods [25], [26], the Takagi-Sugeno results in the output of a control function for the system depending on the values of the inputs. The application of the Sugeno method allows piecewise refinement of a linear relationship of the form that appears in the rule's consequent. However, the linear dependence of each rule on the input variable of a system makes the Sugeno method ideal for acting as an interpolation supervisor of multiple linear controllers that are to be applied, respectively, to different operating conditions of a dynamic nonlinear system as in the present case. The output of the planner is the angle to a free direction. This goes to the second stage to control the robot direction, described by five membership function (MF) as shown in table (1)

Table 1

The fuzzy membership function for the output of the Sugeno-type fuzzy motion planner

\begin{tabular}{|l|l|l|l|}
\multicolumn{4}{|c|}{ motion planner } \\
\hline \hline MF Name & Type & Value & Description \\
SL & Const. & 0.523 & Small to the left $\left(30^{\circ}\right)$ \\
SR & Const. & 0.785 & Big to the left $\left(45^{\circ}\right)$ \\
BR & Const. & -0.523 & Small to the right $\left(30^{\circ}\right)$ \\
F & Const. & -0.785 & Big to the right $\left(45^{\circ}\right)$ \\
GOAL & Const. & 0 & Forward (no change) \\
& Linear & $4^{\text {th }}$ input & $\begin{array}{c}\text { The same angle to the } \\
\text { goal as the } 4^{\text {th }} \text { input }\end{array}$ \\
\hline
\end{tabular}

\section{MODIFIED PROPORTIONAL NAVIGATION BASED FUZZY LOGIC CONTROLLER}

The second stage is an intelligent controller able to optimize the robot's behavior in real time, i.e. to avoid stationary and moving obstacles in its local environment obeying kinematics constraints.

Thus, for the robot to reach the goal, it has to follow two behaviors: (1) when there are no obstacles in the goal sight and (2) when there is an obstacle. Thus, it has to change direction to avoid the obstacle taking into account the dimensions and the kinematics of the robot. In addition, for the general case, a moving goal may also be considered.

To meet these criteria, a fuzzy logic controller (FLC) based on the proportional navigation method was simulated. The proportional navigation method is a guidance law first used in 1950 [27]. It is popular due to its simplicity, effectiveness and ease of implementation [28]

The proportional navigation guidance law seeks to null the line of sight changing rate (LOS) by making the controlled system (robot) turn rate be directly proportional to the rate of turn of sight line [29]. In other words, it seeks to nullify the angular velocity of the line of sight (LOS) angle

In addition to the proportional navigation behavior of the controller, the direction control behavior of the robot in response to an obstacle was also incorporated to give an intelligent behavior fuzzy controller

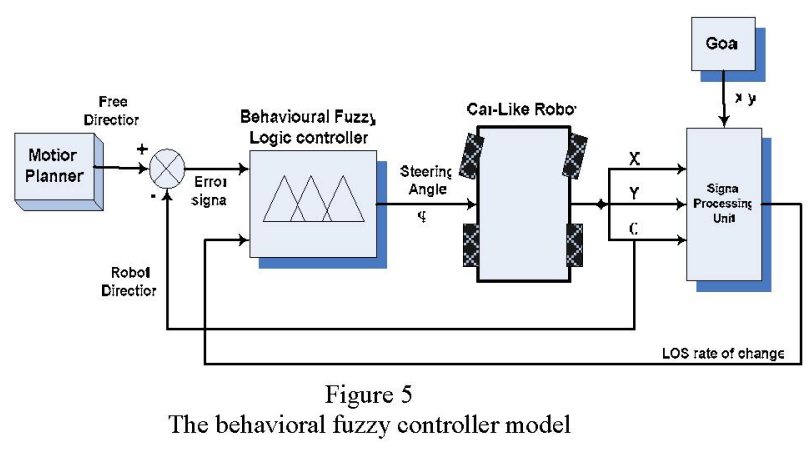

Fig.(5) shows the proposed system model which consists of a two inputs-one output fuzzy controller as shown in Fig.(6).

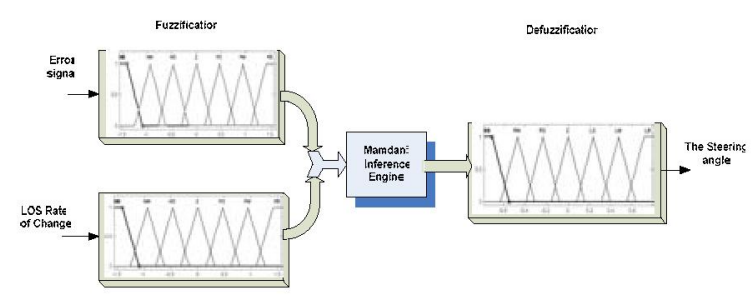

Figure 6

The proposed Behavioral Fuzzy Logic Controller with its Membership Functions

Each crisp input into a fuzzy system can have multiple membership functions assigned to it. In general, the greater number of membership functions assigned to describe an input variable, the higher resolution of the resultant fuzzy control system, resulting in a smoother control response. However, a large number of membership functions requires added computation time and increase the system complexity. Moreover, an excessive number of membership functions can lead to unstable fuzzy system. As a result, the most common number of membership functions for each variable in a fuzzy system fall between 3 and 9 . The number should usually (but not always) be an odd number $(3,5,7,9)$. The control surface fuzzy sets on each side of the zero (or normal) action set should normally be balanced and symmetric

The error signal between the desired direction from the planner and the feedback actual direction of the robot is fuzzified by seven membership functions; five triangle membership functions, and two trapezoidal membership functions. They are used at the inputs to convert the direction error signal into linguistic variables. The trapezoidal membership functions at the boundaries of the desired input range serve to provide "saturation" behavior whenever the input is high. In order to optimize the membership function width the Whole Overlap, WO, index is calculated from the next equation [12]:

$$
W O=\frac{\int_{x} \min \left(\mu_{1}(x), \mu_{2}(x)\right)}{\int_{x} \max \left(\mu_{1}(x), \mu_{2}(x)\right)}
$$

where: $\mu_{1}(\mathrm{x})$ and $\mu_{2}(\mathrm{x})$ are two adjacent membership functions.

A low WO (about 14\%) improved the steady state error and response time [12]. Similarly, the LOS changing rate is fuzzified by seven membership functions. This signal is 
calculated using the proportional navigation concepts [29], $[30]$, in the signal processing unit shown in Fig.(2) as follows:

Calculate the distance and the direction to the goal:

$$
\begin{aligned}
& X_{\text {to_Goal }}=X_{\text {Goal }}-X_{\text {Robot }} \\
& Y_{\text {to_Goal }}=Y_{\text {Goal }}-Y_{\text {Robot }} \\
& \psi_{\text {to_Goal }}=\tan ^{-1} \frac{Y_{\text {to_Goal }}}{X_{\text {to_Goal }}} \\
& R_{\text {to_Goal }}=\sqrt{X_{\text {to_Goal }}^{2}+Y^{2} \text { to_Goal }}
\end{aligned}
$$

Calculate the LOS rate of change:

$$
\text { LOS rate of change }=\frac{\operatorname{vsin}\left(\psi_{\text {to_Goal }}-\theta_{\mathrm{R}}\right)}{\mathbf{R}_{\text {to_Goal }}}
$$

where:

$\left(\mathrm{X}_{\text {Goal, }}, \mathrm{Y}_{\text {Goal }}\right) \quad$ The Goal coordinates

( $\left.\mathrm{X}_{\text {Robot }}, \mathrm{Y}_{\text {Robot }}\right) \quad$ The current robot coordinates

$\left(X_{\text {to_Goal, }} Y_{\text {to Goal }}\right)$ The difference between the robot and Goal coordinates

$$
\begin{array}{ll}
\psi_{\text {to_Goal }} & \begin{array}{l}
\text { The direction to the Goal from the } \\
\text { robot }
\end{array} \\
\mathrm{R}_{\text {to Goal }} & \text { The distance to Goal from robot }
\end{array}
$$

The output of the controller is the steering angle to the robot which is constrained to the range $-45^{\circ}$ to $45^{\circ}$. As with the input variables, seven membership functions are used to represent the output signal. The FLC uses the Mamdani method for the inference engine [31], and the rules are designed such that they combine two behaviors. Firstly when there is no obstacle, the robot behaves in "Track the proportional navigation path until the goal is reached" mode. Secondly, when there is an obstacle, the robot operates in "Deviate from the calculated path and go around the obstacle until it is clear to the goal" mode. These two behaviors are independent therefore they can be developed and demonstrated independently of each other. Thus, each behavior has rules represented in the controller. But, only one action command can be sent to a single output control for any given stimulus (a single point in the input space). This leads to the concept that one behavior must dominate in any given region of the input space. Thus, these two behaviors are merged to create a more complex reasoning system. Thus, these two behaviors are merged to create a more complex reasoning system. The rules were formulated one by one, then the whole rules set was analyzed to make it :

Complete: any combination of the inputs fired at least one rule.

Consistent: it does not contain any contradictions.

Continuous: it does not have neighboring rules with output fuzzy sets that have an empty intersection.

The performance of the fuzzy controller is shown in Fig.(7)

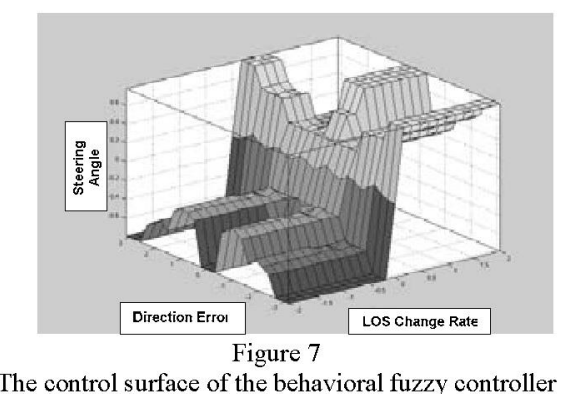

V

\section{RESULTS DISCUSSION AND CONCLUSION}

The system was modeled and simulated with Simulink ${ }^{\mathrm{TM}}$ software [32], for a given data set of random environments. Different environments with different obstacle distributions are used to test the system performance as shown in Fig.(8). The simulation reveals that the system gives good results for the non-complicated environment. This is predictable because of the robot's kinematics constraints.

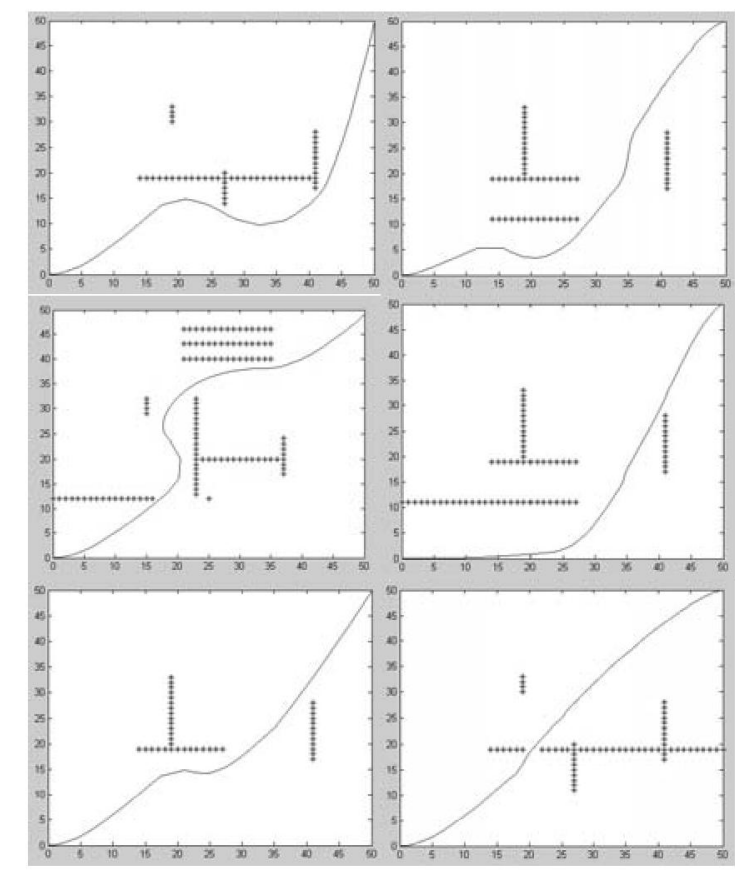

Figure 8

The simulation results for different environments with various obstacle distributions

A number of different strategies has been published to table similar problems. However, the proposed system is compared with similar published systems [13], [9], [10], [33-37]. Different strategies have been used: the behavioral based structure has been used in many [33-37]. These assign different behaviors for different situations (avoiding obstacles, following wall, going to the goal,... etc) and employ a behavior selection mechanism. Such strategies need more training and additional algorithms for tuning the fuzzy parameters. Moreover, the more behaviors we employ the more complex the system becomes. Hierarchical architectures also result in complex system designs [16], [38], [39]. 
By contrast, a real time navigator for a nonholonomic car-like robot in a dynamic environment is proposed. Here in, the system consists of a Sugeno-type fuzzy motion planner and a modified proportional navigation based fuzzy controller. The system philosophy is inspired by human routing when moving between obstacles based on visual information including the right and left views and he makes already the next step to the goal in the free space.

A Sugeno-type fuzzy motion planner of four inputs one output is introduced to give a free direction to the robot controller. A modified proportional navigation based fuzzy logic controller for real time navigation of a nonholonomic car-like robot in a dynamic environment is proposed. It intelligently companies two behaviors to cope with obstacle avoidance as well as approaching a goal using a proportional navigation path accounting for car-like robot kinematics. Other behaviors can be integrated to perform more complex tasks

The main advantage of the present author's system is the simplicity of design which makes it suitable for hardware implementation and extensibility as it does not rely on any specific robotic platform. The system uses a small number of sensors. The system uses linguistic representation which allows the capture of human experiences and intuitive reasoning. In addition, the use of proportional navigation method enables the system to track the goal even if its coordinates have been changed during the navigation which widens the range of proposed system applications.

\section{REFERENCES}

[1] C. Eldershaw, Heuristic Algorithms for Motion Planning, D.Phil Thesis, The University of Oxford, 2001.

[2] G. Song, and N. M. Amato, "A Motion-Planning Approach To Folding: From Paper Craft to Protein Folding", IEEE Transactions on Robotics and Automation, Vol. 20, No. 1, February 2004

[3] H. Zhang and J. P. Ostrowski, "Visual Motion Planning for Mobile Robots", IEEE Transactions on Robotics and Automation, Vol. 18, No. 2, April 2002, pp 199-208.

[4] X. Yang, M. Moallem, and R. V. Patel, "A Layer Goal-Oriented Fuzzy Motion Planning Strategy for Mobil Robot Navigation", IEEE Transactions on System, Man, and Cybernetics, Vol. 35, No. 6, December 2005, pp 1214-1224.

[5] C. Ahrikencheikh and A. Seireg, Optimized-Motion PlanningTheory and Implementation, John Wiley \& Sons Inc., 1994

[6] Y. K. Hwang, and N. Ahuja, "Gross Motion Planning - A Survey", ACM Computing Surveys, Vol.24, No.3, Sept. 1992. pp.219-291

[7] S. M. LaValle, Planning Algorithms, Cambridge University Press, 2005.

[8] B. K. Ghosh, Ning Xi and T. j. Tarn, Control in Robotics and Automation: Sensor-Based Integration, Academic Press, 1999.

[9] Tzuu-Hseng S. Li, M.-S. Chiang and S.-S. Jian, 2000. "Motion planning of an autonomous mobile robot by integrating Gas and fuzzy logic control," Proc. of FUZZ-IEEE-2000, pp. 933-938, May 2000, San Antonio, Texas, USA. (NSC89-2212-E006-063)

[10] H. Surmann, J. Huser and J. Wehking, "Path Planning for a Fuzzy Controlled Autonomous Mobile Robot" 5th IEEE Int. Conf. on Fuzzy Systems FUZZ-IEEE'96, NEW ORLEANS, Sept. 1996.

[11] C. Son "Intelligent control planning strategies with neural network/fuzzy coordinator and sensor fusion for robotic part macro/micro-assembly tasks in a partially unknown environment", International Journal of Machine Tools \& Manufacture, No. 44, 2004, pp. 1667-1681.

[12] L. Reznik, Fuzzy controllers, Newnes, 1997.

[13] N. H. C. Yung and C. Ye, "An intelligent Navigator for Mobile Vehicles" Proceedings of the International Conf. on Neural Information Processing, Hong Kong, 1996, pp. 948-953.

[14] A. Riid and E. Rüstern "Fuzzy Logic in Control : Truck BackerUpper Problem Revisited", Proceedings of the 10th IEEE
International Conference on Fuzzy Systems, Melbourne, Australia, Dec. 2005, pp. 513-516

[15] F. Lamiraux and J.-P. Laumond "Smooth Motion Planning for CarLike Vehicles", IEEE Transactions on Robotics and Automation, Vol.17, No.4, Aug. 2001, pp. 100-104

[16] W. S. Lin, C. L. Huang and M. K. Chuang, "Hierarchical Fuzzy Control for Autonomous Navigation of Wheeled Robots", IEE Proceedings - Control Theory and Applications, September 2005, Vol. 152, Issue 5, pp. 598-606.

[17] G. Oriolo, A. De Luca, and M. Vendittelli, "WMR Control via Dynamic Feedback Linearization: Design, Implementation, and Experimental Validation", IEEE Trans. Control Syst. Technol.,Vol. 10, No. 6, 2002, pp. 835-852

[18] J. M.Yang, and J.H. Kim, "Sliding Mode Control for Trajectory Tracking of Nonholonomic Wheeled Mobile Robots", IEEE Trans. Robot. Autom., Vol.15, No. 3, 1999, pp. 578-587.

[19] R. Fierro and F.L.Lewis, "Control of $A$ Nonholonomic Mobile Robot Using Neural Networks", IEEE Trans. Neural Netw., Vol. 9, No. 4, 1998, pp. 589-600.

[20] R. Colbaugh, E. Barany, and K.Glass, "Adaptive Control of Nonholonomic Robotic Systems", J. Robot. Syst.,Vol.15, No.7, 1998, pp. 365-393.

[21] K. Gupta, and A. P. Pobil, Practical Motion Planning in Robotics: Current approach and Future Directions, John Wiley \& Sons Ltd, 1998.

[22] Advanced Electronics for Robotics, http://www.robotelectronics.co.uk.

[23] K. Walker and A. C. Esterline, "Fuzzy motion planning using the Takagi-Sugeno method', Southeastcon 2000, Nashville, TN, USA, Proceedings of the IEEE, April 2000, pp.56 - 59.

[24] D. Tsay, H.-Y. Chung, and C.-J. Lee, "The Adaptive Control of Nonlinear Systems Using the Sugeno-Type of Fuzzy Logic", IEEE Transactions On Fuzzy Systems, Vol. 7, No. 2, April 1999, pp $225-$ 229.

[25] A. M. Ibrahim, Introduction to Applied Fuzzy Electronics, Prentice Hall Inc., 1997.

[26] J. J. Buckley and E. Eslami, An Introduction to Fuzzy Logic and Fuzzy Sets, Phydica-Verlag Heidelberg, 2002.

[27] V. Rajasekhar and A. G. Sreenatha "Fuzzy Logic Implementation of Proportional Navigation Guidance", Acta Astronautica, Vol. 46, No.1, 2000, pp. 17-24.

[28] M. Mehrandezh, M. N. Sela, R. G. Fenton, and B. Benhabib, "Robotic interception of moving objects using ideal proportional navigation guidance technique" Journal of Robotics and Autonomous Systems, Vol.28, 1999, pp 295-310.

[29] S. A. Murtaugh, and H. E. Girel, "Fundamental of Proportional Navigation", IEEE Spectrum, December, 1966, pp.75-85

[30] J. M. Borg, An Industrial Robotic System For Moving Object Interception Using Ideal Proportional Navigation Guidance, Master Thesis, University of Toronto, 2000.

[31] S. S. Farinwata, D. Filev, and R. Langari, Fuzzy Control : Synthesis and Analysis, John Wiley \& Sons Ltd, 2000.

[32] W. C. Messner and D. M. Tilbury, Control Tutorials for Matlab and Simulink, Addison-Wesley Publishing Company, Inc., 1998.

[33] V. K.Hombal, A. S. Sekmen, and M. S. Zein-Sabatto, "A Fuzzy integrated Robotic Behavioral Architecture", Proceeding of the IEEE Southeastcon conf., Nashville, TN, 2000

[34] H. Seraji, and A. Howard, "Behavior-Based Robot Navigation on Challenging Terrain: A Fuzzy Logic Approach", IEEE Trans. on Robotics And Automation, Vol. 18, No. 3, 2002, pp. 308-321.

[35] M. Wang, and J. N. Liu, "Automatic Robot Navigation Using Fuzzy Logic Controller", Proceeding of the $3^{\text {rd }}$ International Conference on Machine Learning and Cybernetics, Shanghai, 2004.

[36] A. António and L. Correia., "A fuzzy behovior-based architecture for decision control in autonomous vehicles", Proceedings of the 2001 IEEE International Symposium on Intelligent Control ISIC'01, Mexico-city, Mexico, 2001.

[37] S. X. Yang, H. Li, M. Q.-H. Meng, and P. X. Liu, "An Embedded Fuzzy Controller for a Behavior-Based Mobile Robot With Guaranteed Performance", IEEE Transactions on Fuzzy Systems, Vol. 12, No. 4, 2004

[38] I. Baturone, F. J. Moreno-Velo, S. Sánchez-Solano, and A. Ollero, "Automatic Design of Fuzzy Controllers for Car-Like Autonomous Robots" IEEE Transactions on Fuzzy Systems, Vol. 12, No. 4, 2004.

[39] H. A. Hagras, "A Hierarchical Type-2 Fuzzy Logic Control Architecture for Autonomous Mobile Robots", IEEE Transactions on Fuzzy Systems, Vol. 12, No. 4, 2004. 